\title{
Soil Densification Effect on The Seismic Response of Structures Taking into Consideration Soil-Structure Interaction
}

\author{
Radhwane Boulkhiout ${ }^{*}$, , Salah Messast ${ }^{2}$ \\ ${ }_{1,2}^{1}$ Laboratory of Materials, Geotechnical, Habitat and Urbanism (LMGHU), Department of Civil Engineering, University of 20 Aout \\ 1955-Skikda, 21000, Algeria
}

\section{Keywords}

Compaction techniques, Soilstructure interaction,

Impedances functions, CONAN, Cone method,

Lateral displacement, Equivalent static method.

\begin{abstract}
Soil compaction is a considerable construction activity to ensure safety and durability, notably in the transportation industry. This technique of compaction increases soil bulk density and soil strength, while decreases porosity, aggregate stability index, soil hydraulic conductivity, and nutrient availability, thus reduces soil health. Consequently, it lowers crop performance via stunted aboveground growth coupled with reduced root growth. Therefore, if the characteristics of the soil are changed, it will affect the response of the structures. In this work, the effect of improving soil characteristics by compaction techniques on the dynamic response of foundations and structures, taking into consideration the effect of soilstructure interaction was determined. The dynamic response of foundations is presented by the impedances functions, which are determined numerically by the CONAN program, based on the cone method. In addition, the response of the structure will be presented according to the lateral displacement in each level of it. This motion vector is a function of the forces in each level; for this, the equivalent static method was applied, which allows to calculate the seismic force at the base and its distribution on the height of the structure. The results obtained show the efficiency of soil densification on the seismic response of MDOF frames.
\end{abstract}

\section{Introduction}

Compaction techniques (otherwise known as mass densification) are now widely used techniques to improve certain soil characteristics significantly $[1,2,3]$. More specifically, dynamic compaction, which consists of improving the mechanical properties of the soil by transmitting high energy impacts to the loose soils, which initially have a low bearing capacity and high compressibility potentials, which produces the body and the surface waves propagating through the middle of soil. In unsaturated soils, the waves move the grains and rearrange them into a more dense configuration. In saturated soils, the soil is liquefied and the grains rearranged into a more compact state. In both cases, decreasing voids and increasing internal granular contact will directly result in improved soil properties, as shown in Figure 1.

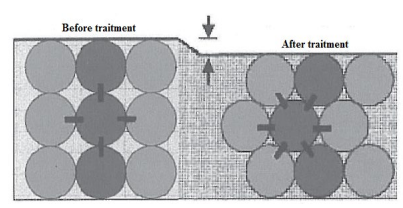

Figure 1. Displacement of soil grains under the effect of dynamic compaction

The foundations considered as elements of contact between the soil and the structure. They have played the role of intermediary between the upper and the lower parts; it allows the transformation of the loads of the structures on the soil or the opposite, because of that the foundations must be well dimensioned for achieving this objective. In the literature, the dynamic response of foundations presented by the impedances functions [4,5]. These functions using in rheological modeling by springs and dampers at the base of structures [6], for which the movement of a foundation in any direction will impose.

The earthquake considered one of the most complex phenomena in soils, because of its random propagation in the soil, which makes it difficult to adapt. The equivalent static method considered a one of the methods used in the seismic analysis of structures, which allows calculating the seismic force at the base of the structure as shear force, and the distribution of it at each level of the structure as static forces [7].

The aim of this study was to investigate the impact of the soil compaction on the lateral displacement of MDOF structures with considering soil-structure interaction effect by a new procedure of substructure method.

\section{Reference model}

The structure is three degrees of freedom reinforced concrete frame, each level has a $5 \mathrm{~m}$ long span with a section of $\left(40 \times 70 \mathrm{~cm}^{2}\right)$. Column height is equal to $h=4 \mathrm{~m}$ with a section size of $\left(40 \times 40 \mathrm{~cm}^{2}\right)$. The structure rests on two foundations $\left(2 \times 2 \mathrm{~m}^{2}\right)$. It has the characteristics shown in the Table 1.

Table 1. Parameters of the structure

\begin{tabular}{ccccc}
\hline$\rho_{c}\left(\mathrm{~g} / \mathrm{cm}^{3}\right)$ & $f_{c k}(\mathrm{GPa})$ & $E_{c}(\mathrm{GPa})$ & $v_{c}$ & $\xi_{c}$ \\
\hline 2.5 & 0.035 & 25 & 0.2 & 0.05 \\
\hline
\end{tabular}

The frame rests on two square foundations $\left(2 \times 2 \mathrm{~m}^{2}\right)$, founded on four types of seating soil (see Figure 2 ) with the parameters given in Table 2.

Table 2. Parameters of seating soil before and after compaction

\begin{tabular}{lccc}
\hline Soil condition & Soil type & $\mathrm{V}_{\mathrm{s}}(\mathrm{cm} / \mathrm{s})$ & $\begin{array}{c}\rho_{\mathrm{s}} \\
\left(\mathrm{g} / \mathrm{cm}^{3}\right)\end{array}$ \\
\hline Before compaction & $\mathrm{E}$ & 17000 & 1.8 \\
After compaction & $\mathrm{D}$ & 18500 & 2.0 \\
Before compaction & $\mathrm{D}$ & 30000 & 2.0 \\
After compaction & $\mathrm{C}$ & 36500 & 2.2 \\
\hline
\end{tabular}


Shear modulus and elasticity modulus of the soil are defined by Equations (1) and (2), respectively.

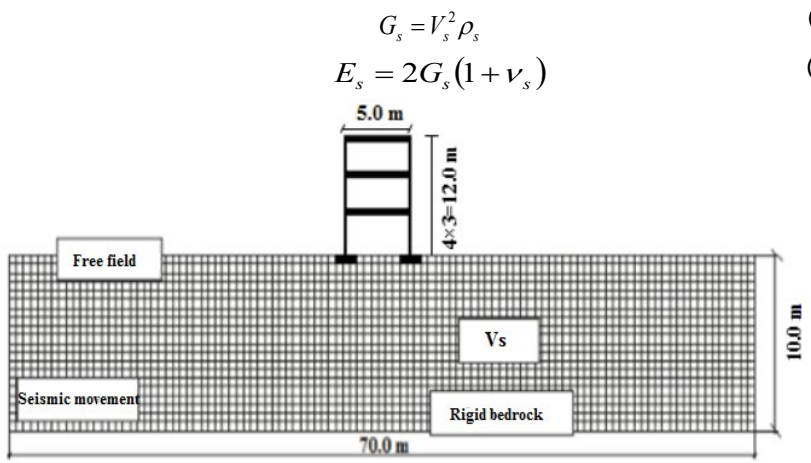

Figure 2. Dimensions of reference model

\section{Calculation method}

The semi-analytical method used in this work combining two methods one numerical allowing to calculate the dynamic impedances of foundation by CONAN program, and the other analytical allowing to calculate the seismic forces in each level of the frame by the equivalent static method ESM. This method makes it possible to calculate the vector of the lateral movement of the three degrees of freedom frame, which considered free at the base as defined by Equation 3:

$$
\left\{\begin{array}{l}
\overline{\Delta_{1}} \\
\overline{\Delta_{2}} \\
\overline{\Delta_{3}}
\end{array}\right\}=\frac{1}{K}\left\{\begin{array}{l}
F_{1} \\
F_{2} \\
F_{3}
\end{array}\right\}\left[\begin{array}{lll}
1 & 1 & 1 \\
1 & 2 & 2 \\
1 & 2 & 3
\end{array}\right]+\frac{1}{K_{h}}\left\{\begin{array}{l}
F_{1} \\
F_{2} \\
F_{3}
\end{array}\right\}+\frac{1}{K_{\phi}}\left\{\begin{array}{l}
F_{1} \cdot h_{1}^{2} \\
F_{2} \cdot h_{2}^{2} \\
F_{3} \cdot h_{3}^{2}
\end{array}\right\}
$$

\subsection{Cone method}

The cone model generally used to determine the values of the rheological models considered in the modeling of subdomain by the substructures method. In this model, the components of the displacement field will vary along the depth in the shape of a truncated cone, as shown in Figure 3, for the horizontal translational degree of freedom [8].

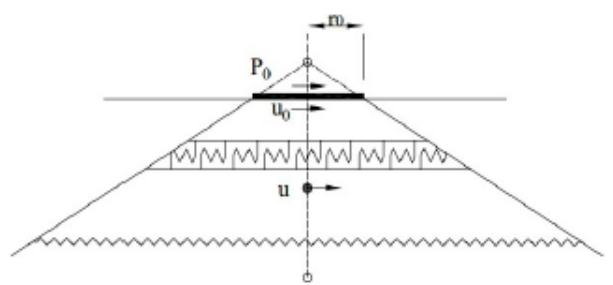

Figure 3. Cone model

One of the numerical programs based on the cone model is the CONAN tool, which allows to determine the static stiffnesses, the stiffness coefficient and the damping coefficient as a function of the dimensionless frequency. For example, in the case of applying a horizontal force to a foundation placed on a homogeneous medium, the terms of the dynamics impedances are given by Equation 4 as follows:

$$
\left\{\begin{array}{l}
k_{s}=\frac{G_{s} \pi r_{0}}{\cot (\alpha)} \\
k_{1}=1 ; \forall a_{0} \\
c_{1}=\cot (\alpha) ; \forall a_{0}
\end{array}\right.
$$

\subsection{Equivalent static method}

The equivalent static method is a simplification technique, makes it possible to replace the dynamic calculation under the effect of a seismic excitation at a force distributed laterally on a structure (see Figure 4) [9]. The total seismic force at the base given by Equation 5 as follows:

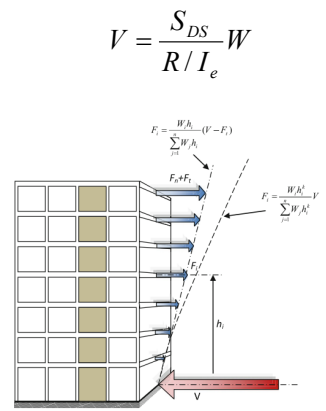

Figure 4. Vertical distribution of the lateral static force

\section{Results and analysis}

Dynamic impedances are absolutely influenced by the shear wave velocity of the soil. The effect of changing the latter will be determined using densification technique on the values of dynamic impedances using the same previous calculation tool. Figures 5-6 shows the horizontal and rotational impedance variations function of dimensionless frequency respectively.
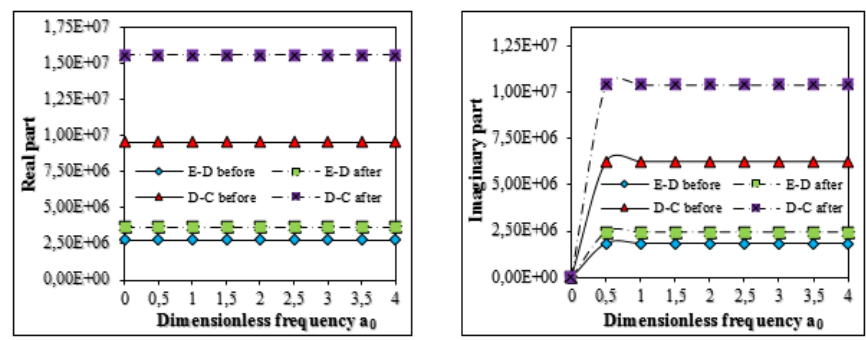

Figure 5. Effect of soil densification on the horizontal impedance
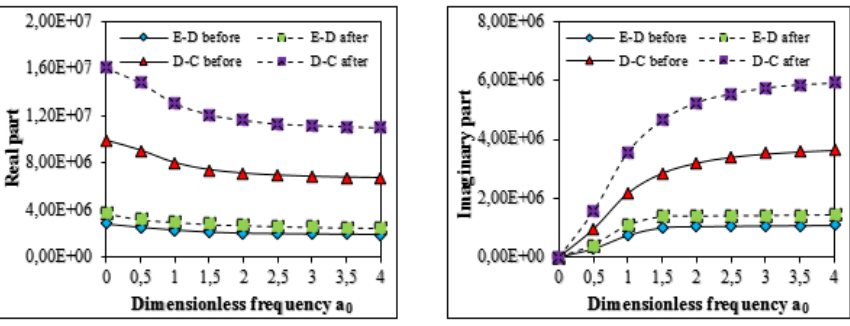

Figure 6. Effect of soil densification on the rotational impedance

The results show the efficiency of densification of the supporting soil on stiffness and damping which are highly affected by its effect. A considerable increase in stiffness and damping in all dynamic impedances was observed. Therefore, compaction has a direct influence on soil damping and stiffness in a proportionate manner.

The effect of the supporting soil on the response of three degrees of freedom frame through dynamic impedances was calculated after compaction of seating soil. Maximum lateral displacements at each level of the frame before and after the densification of the supporting soil are summarized in the Table 3, and are presented in the Figure 7. 
Table 3. Maximum lateral displacements of the frame before and after densification of the supporting soil

\begin{tabular}{lcccc}
\cline { 2 - 5 } & \multicolumn{2}{c}{ Soil E-D } & \multicolumn{2}{c}{ Soil D-C } \\
\cline { 2 - 5 } & Before & After & Before & After \\
\hline $\begin{array}{l}\text { Displacement at } \\
\text { level 1 (cm) }\end{array}$ & 0.210 & 0.200 & 0.198 & 0.181 \\
$\begin{array}{l}\text { Displacement at } \\
\text { level 2 (cm) }\end{array}$ & 0.374 & 0.370 & 0.365 & 0.333 \\
$\begin{array}{l}\text { Displacement at } \\
\text { level 3 (cm) }\end{array}$ & 0.500 & 0.490 & 0.471 & 0.428 \\
\hline
\end{tabular}

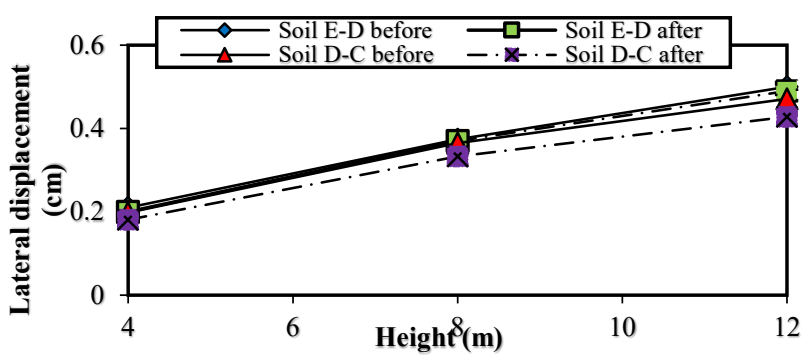

Figure 7. Effect of soil densification on the lateral displacement of the three levels of the frame

The effect of densified soil on the lateral displacement of the three levels of the frame is well illustrated in the figure 7. The lateral displacement of the structure is significantly reduced after the densification of the supporting soil, particularly in the upper level. The temporal variation of this displacement in the three levels of the frame is presented in Figure 8.
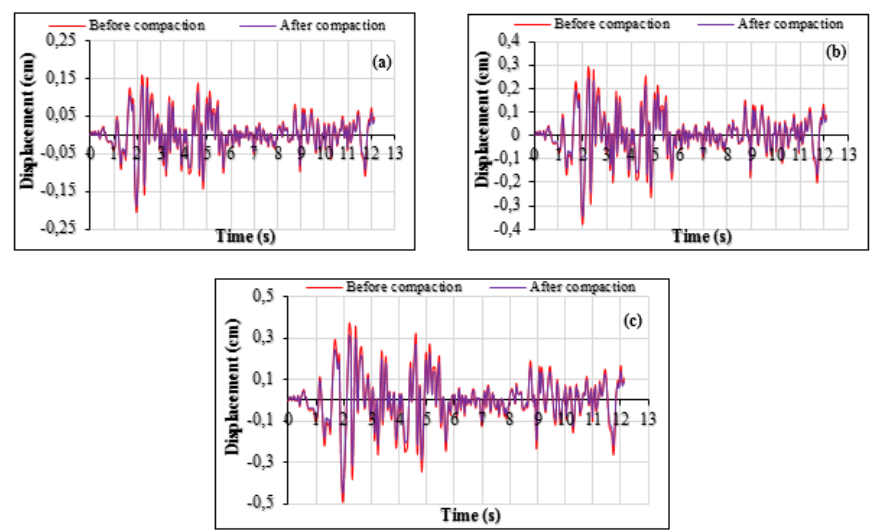

Figure 8. Effect of soil densification on the lateral displacement at: (a) first level, (b) second level, and (c) third level

Figure 8 shows the effect of the densification of the seating soil on the temporal variation of lateral displacement in the three levels of the frame. This effect was greatest in the upper level of the frame when soil acceleration was greatest. Therefore, dynamic compaction increases the shear wave velocity in the soil, which causes a decrease in lateral displacement in all levels of the structure. Table 4 shows the effect of densification technique on the lateral displacement of the three levels of the frame in \%. The obtained results illustrate that the effect of densification is directly proportional to the height of the frame. Which shows it has a relation with the properties of the soil on the one hand and the properties of the structure on the other hand.

Table 4. Effect of compaction on the lateral displacement of the frame

\begin{tabular}{lccc}
\multicolumn{1}{c}{ Level } & 1 & 2 & 3 \\
\hline $\begin{array}{l}\text { Effect of } \\
\text { compaction } \\
(\%)\end{array}$ & 8.58 & 8.77 & 9.13 \\
\hline
\end{tabular}

\section{Conclusion}

In this work, a semi-analytical method was presented, for which we determined the effect of soil densification by dynamic compaction on the lateral displacement of a three degrees of freedom frame. This method combines the cone method with the equivalent static method, which makes it possible to achieve the following points:

- Only the horizontal and the rotational impedances influenced on the lateral displacement of structures.

- The equivalent static method makes it possible to transform the dynamic calculation to a static calculation, for which, the seismic force in the soil was determined as a shear force distributed on the height of the structure.

- The dynamic compaction of supporting soils increases the shear wave velocity in them, which causes an increase of translation and rotation dynamics impedances, as well as a decrease of lateral displacement of the structures.

- Dynamic compaction minimizes the lateral displacement of structures, which gives an economic reserve concerning the dimensioning of the sections.

\section{Nomenclature}

$S_{D S}:$ Acceleration response at short periods

$\alpha$ : Angle

$I_{c}:$ Column inertia

$K_{i}:$ Column stiffness on the $i^{\text {th }}$ floor

$c_{1}:$ Damping coefficient

$a_{0}$ : Dimensionless frequency

$\overline{\Delta_{i}}$ : Lateral displacement of the $i^{\text {th }}$ floor

$E_{c}:$ Elastic modulus of columns

$r_{0}:$ Equivalent radius of foundation

$h_{i}:$ Height of the static forces on the $i^{\text {th }}$ floor

$R:$ Response modification factor

$I_{e}$ : Seismic importance factor ensures a seismic force of a superior design for larger structures

$F_{i}$ : Static forces on the $i^{\text {th }}$ floor

$k_{s}:$ Static stiffnesses

$k_{1}:$ Stiffnesses coefficient

$W:$ Total seismic weights

\section{Declaration of Conflict of Interests}

The authors declare that there is no conflict of interest. They have no known competing financial interests or personal relationships that could have appeared to influence the work reported in this paper. 


\section{References}

[1.] Hamidi, B., Nikraz, H., Varaksin, S., A review of impact oriented ground improvement techniques. Australian Geomechanics Journal 44 (2009) 17-24.

[2.] Orzech, K., Załuski, D., Effect of soil compaction and different soil tillage systems on chemical properties. Journal of Elementology 29(2019) 413-429.

[3.] Borgmann, C., Secco, D., Ciotti de Marins, A., Junior, L. A. Bassegio, D., Effect of Soil Compaction and Application of Lime and Gypsum on Soil Properties and Yield of Soybean. Communications in Soil Science and Plant Analysis 52(2021)1434-1447.

[4.] Lee J.H., Kim J.K., Tassoulas, J.L., Dynamic analysis of foundation in a layered half-space using a consistent transmitting boundary. Earthquake structure 3 (2012) 203-230.

[5.] Borghei, A., Ghayoomi, M., Centrifuge testes to evaluate dynamic impedance functions of square surface foundation. 7th International Conference on Earthquake Geotechnical Engineering, Rome, Italy, 17-20 June, 2019, 1495-1502.

[6.] Chen, Y., Zhao, W., Jia, P., Han, J., Guan, Y., Dynamic behavior of an embedded foundation under horizontal vibration in a poroelastic half-Space. Applied Science 9 (2019) 1-18.

[7.] Koukouselis, A., Chatziioannou, K., Mistakidis, E., Katsardi, V., Development of an equivalent static method for the approximation of the dynamic response of offshore structures. Engineering Computations 36 (2019) 1121-1141.

[8.] Farajian, M., Khodakarami, M.I., Kontoni, D.N., Evaluation of SoilStructure Interaction on the Seismic Response of Liquid Storage Tanks under Earthquake Ground Motions. Computation 5 (2017) 112.

[9.] Bourahla, N., Equivalent Static Analysis of Structures Subjected to Seismic Actions. Encyclopedia of Earthquake Engineering (2013) 1-13.

\section{How to Cite This Article}

Boulkhiout, R., Messast, S., Soil Densification Effect on The Seismic Response of Structures Taking into Consideration Soil-Structure Interaction, Civil Engineering Beyond Limits, 4(2021), 13-16. https://doi.org/10.36937/cebel.2021.004.003 